\title{
Map-based cloning and characterization of Zea mays male sterility 33 (ZmMs33) gene, encoding a glycerol-3-phosphate acyltransferase
}

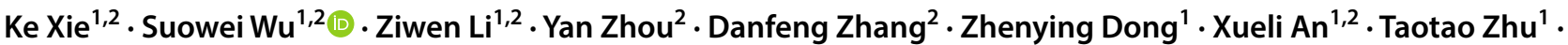 \\ Simiao Zhang ${ }^{1}$. Shuangshuang $\mathrm{Liu}^{2} \cdot$ Jinping $\mathrm{Li}^{2} \cdot$ Xiangyuan Wan ${ }^{1,2} \mathbb{Z}$
}

Received: 10 September 2017 / Accepted: 6 March 2018 / Published online: 15 March 2018

(c) The Author(s) 2018

\begin{abstract}
Key message Map-based cloning of maize $m s 33$ gene showed that $Z m M s 33$ encodes a $s n-2$ glycerol-3-phosphate acyltransferase, the ortholog of rice OSGPAT3, and it is essential for male fertility in maize.

Abstract Genetic male sterility has been widely studied for its biological significance and commercial value in hybrid seed production. Although many male-sterile mutants have been identified in maize (Zea mays L.), it is likely that most genes that cause male sterility are unknown. Here, we report a recessive genetic male-sterile mutant, male sterility33 (ms33), which displays small, pale yellow anthers, and complete male sterility. Using a map-based cloning approach, maize GRMZM2G070304 was identified as the $m s 33$ gene (ZmMs33). ZmMs33 encodes a novel $s n$-2 glycerol-3-phosphate acyltransferase (GPAT) in maize. A functional complementation experiment showed that GRMZM2G070304 can rescue the male-sterile phenotype of the ms33-6029 mutant. GRMZM2G070304 was further confirmed to be the ms33 gene via targeted knockouts induced by the clustered regularly interspersed short palindromic repeats (CRISPR)/Cas9 system. ZmMs33 is preferentially expressed in the immature anther from the quartet to early-vacuolate microspore stages and in root tissues at the fifth leaf growth stage. Phylogenetic analysis indicated that ZmMs33 and OsGPAT3 are evolutionarily conserved for anther and pollen development in monocot species. This study reveals that the monocot-specific GPAT3 protein plays an important role in male fertility in maize, and ZmMs33 and mutants in this gene may have value in maize male-sterile line breeding and hybrid seed production.
\end{abstract}

\section{Abbreviations \\ AT Acyltransferase \\ BLAST $_{\mathrm{P}} \quad$ Basic Local Alignment Searching Tool- protein to protein \\ CD Search Conserved domain search service \\ CMS Cytoplasmic male sterility}

Communicated by Michael Gore.

Ke Xie, Suowei Wu and Ziwen Li have contributed equally to this work.

Electronic supplementary material The online version of this article (https://doi.org/10.1007/s00122-018-3083-9) contains supplementary material, which is available to authorized users.

Xiangyuan Wan

wanxiangyuan@ustb.edu.cn

1 Advanced Biotechnology and Application Research Center, School of Chemistry and Biological Engineering, University of Science and Technology Beijing, Beijing 100024, China

2 Beijing Engineering Laboratory of Main Crop Biotechnology Breeding, Beijing Solidwill Sci-Tech Co. Ltd, Beijing 100192, China
CRISPR Clustered regularly interspersed short palindromic repeats

CTAB Cetyltrimethyl ammonium bromide

ER Endoplasmic reticulum

GMS Genetic male sterility

GPAT Glycerol-3-phosphate acyltransferase

LPA Lysophosphatidic acid

2-MAG sn-2 Monoacylglycerol

MaizeGDB Maize Genetics and Genomics Database

MAS Marker-assisted selection

MCS Multi-control sterility

NCBI National Center for Biotechnology

Information

RGAP Rice Genome Annotation Project

RT-PCR Reverse transcription polymerase chain reaction

TM Transmembrane 


\section{Introduction}

Male sterility refers to cases in which viable male gametes (i.e., pollen) are not produced, while female gametes are fully fertile. In maize, there are two general types of male sterility based on the pattern of inheritance: cytoplasmic male sterility (CMS) and genetic male sterility (GMS). CMS is a maternally inherited inability to produce viable pollen that is associated with novel mitochondrial open reading frames (Cui et al. 1996; Feng et al. 2015; Tan et al. 2011). Although a CMS system has been successfully used for maize hybrid seed production, some severe problems, such as the somewhat unsteady heredity of male fertility under certain environments, the narrow germplasm resources of restore lines, the poor genetic diversity between the CMS lines and restore lines, have restricted extensive application in maize ( $\mathrm{Hu}$ et al. 2006; Kohls et al. 2011; Williams 1995). GMS occurs when lesions in nuclear-encoded genes disrupt normal male gametogenesis. GMS mutants can be either dominant or recessive and typically exhibit Mendelian inheritance (Fox et al. 2017; Tang et al. 2006). The majority of the mutant phenotypes identified to date are controlled by recessive genes, which provide an excellent means of genetic emasculation for hybrid seed production in maize.

GMS is controlled by nuclear genes, mainly those that affect tapetum or microspore development in flowering plants (Jung et al. 2005). Maize is a monoecious crop, which has a separate tassel (male flower) and ear (female flower) on the same plant, making it advantageous for studying anther and pollen development. In maize, anther and pollen development is very complex, with the expression of about 24,000-32,000 genes in a span of nearly 30 days (Ma et al. 2008; Skibbe et al. 2009). Since the first report of a GMS mutant in maize (Eyster 1921), there have been hundreds of maize male-sterile mutants defined from phenotypic scoring (Timofejeva et al. 2013). However, only a handful of these mutants have been characterized cytologically, and even fewer male-sterile genes have been isolated. Most of them are recessive GMS genes, such as ZmMs 7 (Zhang et al. 2018), Ms8 (Wang et al. 2013), $M s 9$ (Albertsen et al. 2016), Ms22/Mscal(Albertsen et al. 2009), Ms23 (Nan et al. 2017), Ms26 (Djukanovic et al. 2013), Ms32 (Moon et al. 2013), Ms45 (Cigan et al. 2001), APVI (Somaratne et al. 2017), IPE1 (Chen et al. 2017), MACl (Kelliher and Walbot 2012; Wang et al. 2012) and OCL4 (Vernoud et al. 2009). To date, Ms 44 has been the only dominant GMS gene identified in maize, and it encodes a lipid transfer protein which is expressed specifically in the tapetum (Fox et al. 2017). The cloning and functional characterization of these male-sterile genes have contributed significantly to our understanding of the molecular mechanisms of anther and pollen development in maize and have provided useful genetic resources for genetic engineering of male-sterile lines for hybrid seed production. Nevertheless, compared with the deep understanding of the molecular mechanisms and gene networks related to anther and pollen development in the model species Arabidopsis and rice (Gomez et al. 2015; Shi et al. 2015), comparatively little is known about the equivalent processes in maize.

Glycerol-3-phosphate acyltransferase (GPAT; EC2.3.1.15) is the key enzyme in the glycerolipid synthetic pathway, catalyzing the first dedicated step in the biosynthesis of membrane lipids, storage lipids, and extracellular lipid polyesters (cutin and suberin) (Fig S1). In general, GPATs use acyl-CoA as a substrate, from which they transfer the acyl chain onto glycerol-3-phosphate. The $s n-1$ GPATs mediate the acylation at the $s n-1$ position of glycerol3-phosphate to produce lysophosphatidic acid (LPA). LPA is an important intermediate for the formation of various types of lipids, such as extracellular lipid polyesters, storage and membrane lipids. Some GPATs possess a phosphatase domain that results in $s n-2$ monoacylglycerol (2-MAG) rather than LPA as the major product. These are named $s n-2$ GPATs, and they are involved in cutin and suberin synthesis in land plants. To date, most GPATs have been found to be sn-2 GPATs (Beisson et al. 2012; Li et al. 2007; Yang et al. 2012).

In the Arabidopsis genome, there are ten GPATs, which can be divided into two families: $s n-1$ and $s n-2$ GPAT. The $s n-1$ GPAT family includes ATS1 and AtGPAT9. ATS1 is a soluble and cytoplasm-localized GPAT that utilizes acylACP substrates and exhibits $s n-1$ acyl transfer regiospecificity (Nishida et al. 1993). AtGPAT9 is an ER-localized GPAT enzyme responsible for plant membrane lipid and oil biosynthesis in developing Arabidopsis seeds and leaves, as well as lipid droplet production in developing pollen grains. The AtGPAT9 knockout mutant demonstrates both male and female gametophytic lethality phenotypes (Shockey et al. 2016). The $s n$-2 GPAT family, which consists of the remaining eight members from AtGPAT1 to AtGPAT8 belonging to a land-plant specific family with three distinct clades, are required for the synthesis of cutin and suberin. AtGPAT4/6/8 comprise the cutin-associated clade and are unique bifunctional enzymes with $s n-2$ acyltransferase and phosphatase activity resulting in 2-MAG products (Yang et al. 2012). The suberin-associated clade includes AtGPAT5/7. AtGPAT5 is an acyltransferase with broad acyl-CoA specificity that is required for root and seed coat suberin biosynthesis. AtGPAT7 exhibits wounding-induced expression, produces suberin-like mononers when overexpressed and likely functions in suberin biosynthesis (Yang et al. 2012). Finally, AtGPAT1/2/3 comprise the third clade. AtGPAT1 possesses $s n-2$ acyltransferase but not phosphatase activity 
and can utilize discarboxylic acyl-CoA substrates (Zheng et al. 2003). However, no acyltransferase activity for AtGPAT2 and AtGPAT3 has been detected, and the corresponding mutants display no obvious phenotypes or changes in their polymeric lipids in flowers, leaves or seeds (Yang et al. 2012).

GPATs play important biological roles in plant pollen and anther development. For example, AtGPAT1 is a membranebound GPAT protein that was found to be important for tapetum differentiation and nutrient secretion. Consequently, disruption of AtGPATl causes abortion of microspores before maturity. AtGPAT6 plays diverse roles in the development of pollen exine and coat, tapetum formation and stamen elongation. Both Arabidopsis gpatl and gpat6 mutants display altered endoplasmic reticulum (ER) profiles in tapetal cells as well as severely reduced pollen production and decreased pollination (Li et al. 2012; Zheng et al. 2003). Similarly, the gpat6 mutant showed disturbed pollen formation in tomatoes (Petit et al. 2016). However, the molecular mechanism of GPATs in monocots is largely unknown. GPATs in chloroplasts are responsible for the selective incorporation of saturated and unsaturated fatty-acyl chains into chloroplast membrane, which is an important determinant of a plant's ability to tolerate chilling temperatures. There was a report on the substrate selectivity and association with chilling tolerance of a rice plastid-localized GPAT (Zhu et al. 2009). Most recently, one member of the rice GPAT family, OsGPAT3, was reported to be involved in anther development and pollen formation (Men et al. 2017). However, none of the GPAT genes in maize have been investigated until now.

In this study, the maize $m s 33$ gene ( $Z m M s 33)$ was isolated by a map-based cloning approach. ZmMs33 encodes a novel GPAT protein in maize. We characterized $Z m M s 33$ at multiple levels, including phenotypic observation of $m s 33$ mutants, gene structure analysis, functional complementation, targeted mutagenesis induced by a CRISPR/Cas9 system, spatio-temporal expression patterns and phylogenetic analysis. ZmMs33 is the first GPAT gene isolated and characterized in maize, and our results will contribute to the understanding of the molecular mechanism of male sterility in maize. More importantly, ZmMs33 and mutants in this gene may have value in maize male-sterile line breeding and hybrid seed production.

\section{Materials and methods}

\section{Plant materials and growth conditions}

$m s 33-6019$ (Stock ID: 228F), ms33-6029 (Stock ID: 228H), $m s 33-6038$ (Stock ID: 228I) and $m s 33-6052$ (Stock ID: 206F) mutants were obtained from the Maize Genetics Cooperation Stock Centre (http://maizecoop.cropsci.uiuc. edu). The two $\mathrm{F}_{2}$ mapping populations were derived from crosses of $m s 33-6029 \times$ Chang7-2 and $m s 33-6038 \times$ Chang72. All the plants were grown in the field in Beijing or Sanya, China. The $\mathrm{T}_{0}$ transgenic plants and their progeny were grown in a greenhouse in Beijing, China.

\section{Characterization of mutant phenotypes}

Based on the methods as described previously (Zhang et al. 2018), tassels and spikelets were photographed, pollen grains were stained with $1 \% \mathrm{I}_{2}-\mathrm{KI}$ and photographed. Anther staging was defined as described by Zhang et al. (2018). SEM of anthers at stage 13 was performed as described by Chen et al. (2017). Anther staging was defined as described by Albertsen and Phillips (1981).

\section{Map-based cloning}

Genomic DNA was extracted from maize leaves using the CTAB method with some modifications (Wan et al. 2006). As the ms33 locus was mapped to the long arm of maize chromosome 2 (2L) by using B-A translocations (Patterson 1995) and RFLP markers (Trimnell et al. 1999), nine SSR primer pairs on maize chromosome $2 \mathrm{~L}$ were chosen for $m s 33$ primary mapping (Table $\mathrm{S} 1$ ). For fine mapping, several InDel markers (Table S1) were designed with DNAMAN6.0 (LynnonBiosoft). All PCR primers for these markers were synthesized by Sangon Biotech (Shanghai, China) and tested to identify polymorphic markers distinguishing fertile from sterile plants. By scoring the presence/absence of recombinants at diverse marker locations (Wan et al. 2008), the $m s 33$ gene was narrowed down to a $349-\mathrm{kb}$ interval on chromosome $2 \mathrm{~L}$.

\section{Plasmid construction for functional complementation and CRISPR/Cas9 mutagenesis}

For function complementation, the ZmMs33 gene native promoter (1794 bp, Fig. S5) was amplified from maize B73 using primer pair Ms33-ProP, and the ZmMs 33 coding DNA sequence (1578 bp, Figs. S6 and S7) was amplified from the cDNA of B73 anthers using primer pair Ms33-CDSP. The two fragments were fused together with HindIII/BamHI and then subcloned into $p B C X U N$ (Chen et al. 2009) with the hygromycin coding region replaced by an herbicide resistance marker (Bar). The resultant vector was named pZmMs33pro::ZmMs33. For site-directed mutagenesis of ZmMs33, three types of CRISPR/Cas9 plasmids were constructed based on the pBUE411 vector as described previously (Xing et al. 2014). For assembly of one gRNA, the double-stranded short DNA was annealed from two complementary oligos of Cas9-1g-1 (Table S2) and inserted into the pBUE411 vector by $B s a \mathrm{I}$ digestion. The colonies were 
confirmed by sequencing and the corresponding vector was named as pCas9-1g (Fig. 6). For assembly of two gRNAs, PCR fragments were amplified from pCBC-MT1T2 with primers sets (Table S2) among which target sites were incorporated, and then, the purified PCR fragments were digested with $B s a \mathrm{I}$ and cloned into the pBUE411 vector. The colonies were confirmed by sequencing and the corresponding vectors were designated as pCas9-2g-1 or pCas9-2g-2 (Fig. 6).

\section{Maize genetic transformation}

All constructs were transformed into the maize Hi-II hybrid line using the method as described previously (Zhang et al. 2018). The Bar gene was employed as a selectable marker and the transformants were screened by PCR amplification using primer pair Bar-P. The transgenic T-DNA region was then transferred into the $m s 33-6029$ mutant by crossing and backcrossing. To confirm the presence of the ms33-6029 allele and the transgenic T-DNA region in the progeny, the transgenic plants were screened by PCR amplification using primer pairs ms33-ID and Bar-P. All primers described above are listed in Table S2.

\section{Protein alignment and phylogenetic analysis}

Sixty homologues of ZmMs33 were obtained by a Basic Local Alignment Searching Tool-protein to protein $\left(\mathrm{BLAST}_{\mathrm{P}}\right)$ search on the National Center for Biotechnology Information (NCBI), Rice Genome Annotation Project (RGAP) and Maize Genetics and Genomics Database (MaizeGDB) websites. The phylogenetic tree was generated in MEGA7.0 using the maximum likelihood method (Kumar et al. 2016). The amino acid sequences of maize ZmMs33 and the orthologous proteins in barley, rice, sorghum and millet were aligned using DNAMAN6.0, and the conserved domains were analysed with a Conserved Domain Search Service (CD Search) in the NCBI website.

\section{RNA extraction and expression analysis}

Total maize RNA was isolated using the TRIzol reagent (Invitrogen) from the following maize tissues: anthers during different stages, leaves, roots and immature ears. Total RNA $(1 \mu \mathrm{g})$ was used to synthesize the first-strand cDNA with Superscript III RT (Invitrogen). Semi-quantitative reversetranscription PCR (RT-PCR) analyses were conducted using $1 \mu \mathrm{L}$ cDNA as template. RT-PCR was performed on a Bio-Rad MyCycler Thermal Cycler with a standard threestep protocol, consisting of $94{ }^{\circ} \mathrm{C}$ for 2 min followed by 30 cycles of $94{ }^{\circ} \mathrm{C}$ for $30 \mathrm{~s}, 58{ }^{\circ} \mathrm{C}$ for $30 \mathrm{~s}$ and $72{ }^{\circ} \mathrm{C}$ for $30 \mathrm{~s}$. ZmActinl and GAPDH were used as the internal controls. All primers used for RT-PCR are listed in Table S2.

ZmMs33 GenBank accession number: MF615247.

\section{Results}

\section{Maize $m s 33$ is a single recessive mutant exhibiting complete male sterility}

Two ms33 mutants, ms33-6029 and ms33-6038, were obtained from the Maize Genetics Cooperation Stock
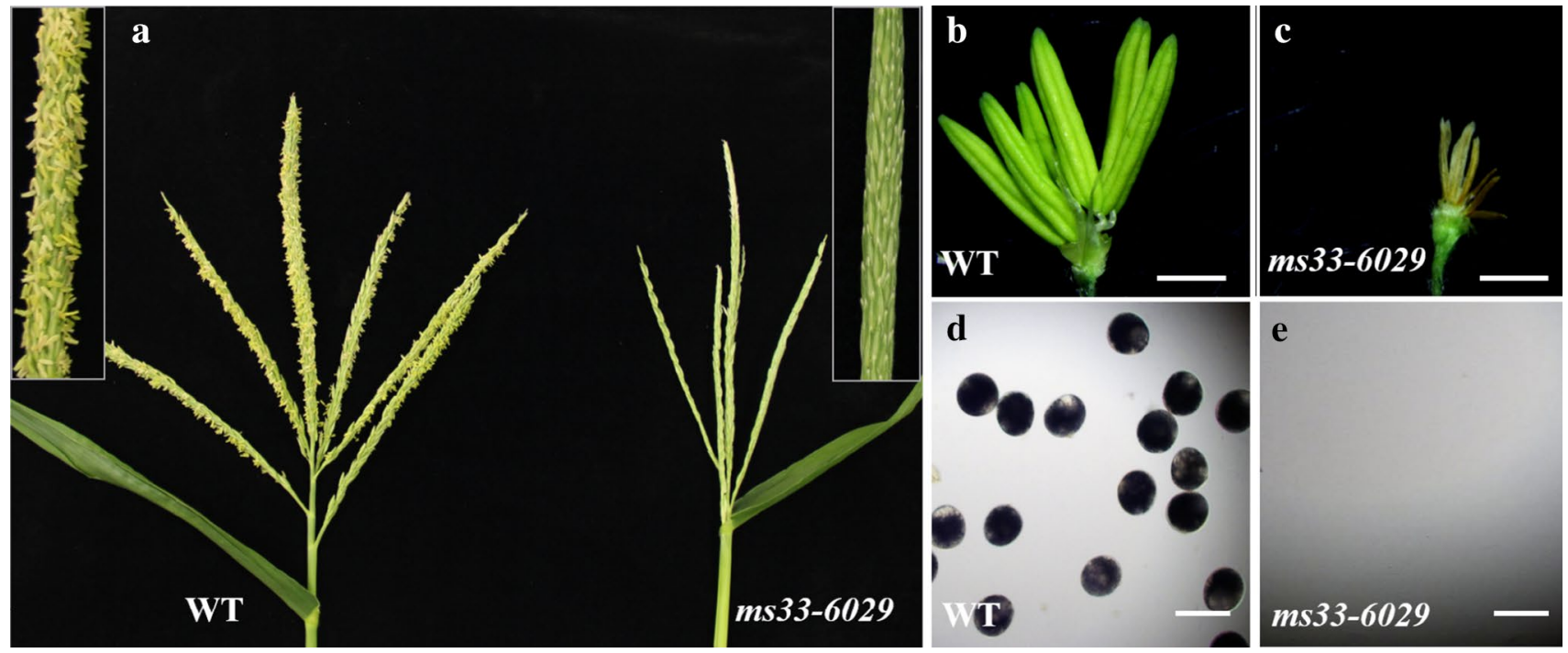

Fig. 1 Phenotypic comparison of the wild type (WT) and ms33-6029 mutant. a The tassels of WT (left) and $m s 33-6029$ (right). b, c The spikelet of WT and $m s 33-6029$ with the glume, lemma and palea removed. d, e The pollen of WT and $m s 33-6029$ stained with $\mathrm{I}_{2}-\mathrm{KI}$. Bars $=2 \mathrm{~mm}(\mathbf{b}, \mathbf{c}), 100 \mu \mathrm{m}(\mathbf{d}, \mathbf{e})$ 
Centre. Compared with the wild-type male-fertile sibling, ms33-6029 displayed complete male sterility with no exerted anthers (Fig. 1a) but normal vegetative growth and female fertility. The mutant anthers were thin and pale yellow (Fig. 1b, c) and lacked pollen grains in the withered anthers, which were not stained with $\mathrm{I}_{2}$-KI (Fig. 1d, e). The mutant phenotype was stable under multiple environments, including different years (2013-2016) and locations (Beijing or Sanya, China, data not shown) (Patterson 1995; Trimnell et al. 1999). The ms33-6038 mutant also showed a similar male-sterile phenotype as the ms33-6029 mutant (Fig. S2).

The two ms33 mutants were crossed with the maize inbred line Chang7-2. All the $\mathrm{F}_{1}$ progeny was male fertile, and the $\mathrm{F}_{2}$ population segregated at a male fertile-to-sterile ratio of 3:1 (Table 1), suggesting that both $m s 33-6029$ and $m s 33-6038$ were single recessive mutations. Furthermore, when the $m s 33-6029 / m s 33-6029$ homozygous plants were outcrossed using pollen from ms33-6038/+ heterozygous plants, the $F_{1}$ progeny showed a 1:1 (101:98) segregation ratio of male sterile to fertile plants, proving that the mutant gene of $m s 33-6029$ is allelic to that of $m s 33-6038$.

\section{Anther and tapetum development was defective in the ms33 mutant}

Scanning electron microscopy (SEM) was used to observe phenotypic differences between the ms33 mutants and wild type at the mature pollen grain stage. Consistent with the above morphological results, the ms33-6029 mutant produced small, wilted anthers with no pollen grains in the anther locule (Fig. 2a-d). A reticulate cuticle coated the outermost surface of anthers, and abundant Ubisch bodies were distributed on the outside of tapetal cells in wild-type anthers (Fig. 2e, g). However, the ms33-6029 anther surface was shrunken and without a reticulate coat (Fig. 2f), indicating that anther cuticle formation was disrupted in the mutant. In addition, Ubisch bodies were not observed on the inner surface of mutant anthers (Fig. 2h). The ms33-6038 mutant also showed similar anther developmental defects

Table 1 The ratio of fertile to sterile plants in the two $\mathrm{F}_{2}$ populations of the ms 33 mutant

\begin{tabular}{llllllll}
\hline $\mathrm{F}_{2}$ population & Total plants & Fertile plants, $F$ & Sterile plants, $S$ & $F / S$ ratio & $\chi^{2}$ & $\begin{array}{l}P \\
\text { cant test, } \\
P>0.05\end{array}$ \\
\hline$m s 33-6029 \times$ Chang7-2 & 916 & 681 & 235 & $2.9: 1$ & 0.087 & 0.7681 & $\mathrm{~ns}$ \\
$m s 33-6038 \times$ Chang7-2 & 966 & 712 & 254 & $2.8: 1$ & 0.3435 & 0.5578 & $\mathrm{~ns}$ \\
\hline
\end{tabular}

ns* means non-significant
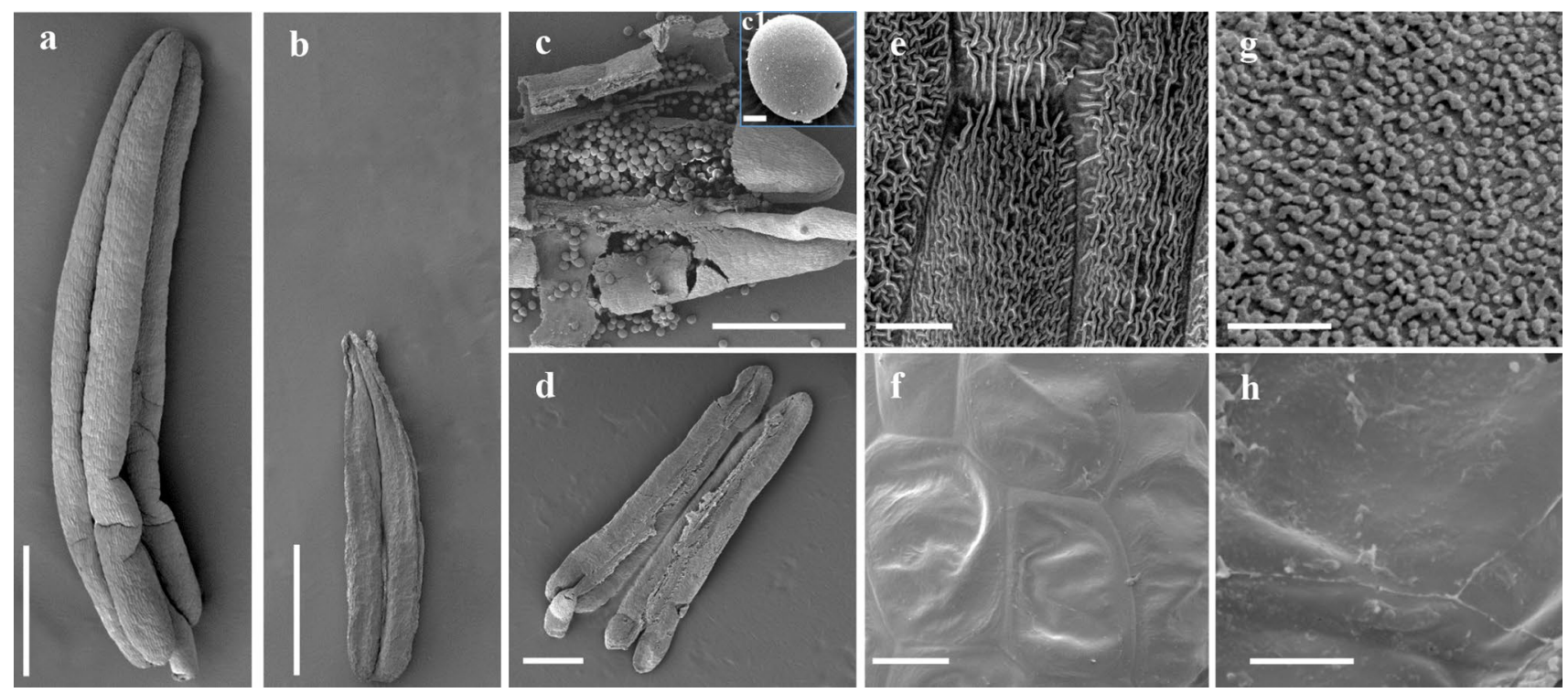

Fig. 2 Appearance of the anther and pollen grain in the wild type (WT) and ms33-6029 mutant at stage 13 of anther development under scanning electron microscopy. a WT and b $m s 33-6029$ anthers. c, c1, d, Pollen grains of WT (c, c1) and the absence of pollen grains in $m s 33-6029$ (d). e, $\mathbf{f}$ The outermost surface of the epidermis of WT (e) and $m s 33-6029$ (f) anthers. $\mathbf{g}, \mathbf{h}$ The inner surface of the anther wall layers of WT (g) and $m s 33-6029$ (h) anthers. Bars $=1 \mathrm{~mm} \mathrm{(a,} \mathrm{b),} 500$ $\mu \mathrm{m}(\mathbf{c}, \mathbf{d}), 15 \mu \mathrm{m}(\mathbf{c 1}), 10 \mu \mathrm{m}(\mathbf{e}, \mathbf{f}), 5 \mu \mathrm{m}(\mathbf{g}, \mathbf{h})$ 
at the same stage (Fig. S3). These results showed that $m s 33$ mutation influences development of the anther cuticle and Ubisch bodies, as well as the formation of pollen grains.

\section{Fine mapping of the $m s 33$ male-sterility gene}

ms 33 was mapped to the long arm of chromosome 2 by B-A translocation and RFLP markers (Patterson 1995; Trimnell et al. 1999). A map-based cloning approach was adopted to isolate the ms 33 mutant gene. Using 228 male-sterile individuals from the $\mathrm{F}_{2}$ population and molecular marker linkage analysis, the $m s 33$ locus was initially mapped between SSR markers EP97 and bnlg1893 on chromosome $2 \mathrm{~L}$ (Fig. 3a). For fine mapping, four InDel markers in the interval were developed (Table S1) and $m s 33$ was narrowed down to a 349-kb interval between the InDel markers EP603 and EP605 (Fig. 3b). According to the annotation from MaizeGDB, fifteen gene models are predicted in this region (Fig. 3c and Table S3), including GRMZM2G070304, is likely to be the $m s 33$ gene based on bioinformatic analysis and sequence comparison between the wild type and two ms33 mutants (see below).

\section{Map-based cloning and functional confirmation of $m s 33$ gene in maize}

GRMZM2G070304 was identified to have two exons and one intron (Fig. 4a) and was likely to encode a putative 525-amino acid GPAT protein, containing a transmembrane
(TM) domain and an acyltransferase (AT) domain (Fig. 4f). Sequencing of this gene in the ms33-6019 mutant revealed that a 479-bp deletion at the +208-686 nucleotide site in the first exon (Fig. 4b), which caused a frame-shift mutation and altered the open reading frame after the 69th amino acid, resulting in a lack of the conserved AT domain (Fig. 4f). Additionally, there were three InDels and five SNPs in the first exon and one SNP in the second exon (Fig. 4b), which resulted in deletion of two amino acids, one amino-acid insertion, and four amino-acid changes (Fig. 4f). Sequencing of this gene in the ms33-6029 mutant revealed that a 1387bp transposable element (DTA_ZM00143) was inserted at the +232 nucleotide site in the first exon (Fig. 4c), which caused a frame-shift mutation and altered the open reading frame after the 77th amino acid, resulting in a lack of the conserved AT domain (Fig. 4f). Additionally, there were two SNPs and a 3-bp deletion in the first exon (Fig. 3c), leading to a single amino-acid change and deletion, respectively (Fig. 4f). Sequencing of this gene in the $m s 33-6038$ mutant revealed a 5-bp (ACACC) deletion at the +572-576 nucleotide site in the first exon (Fig. 4d), which also caused an in-frame premature stop codon and led to deletion of the conserved AT domain (Fig. 4f). Sequencing of this gene in the ms33-6052 mutant revealed a 2-bp (AC) insertion at the +499 nucleotide site in the first exon (Fig. 4e), which also caused an in-frame premature stop codon and led to deletion of the conserved AT domain (Fig. 4f). Similar with the $m s 33-6019$, there were several InDels and SNPs in the first and second exon of GRMZM2G070304 in the ms33-6038

a
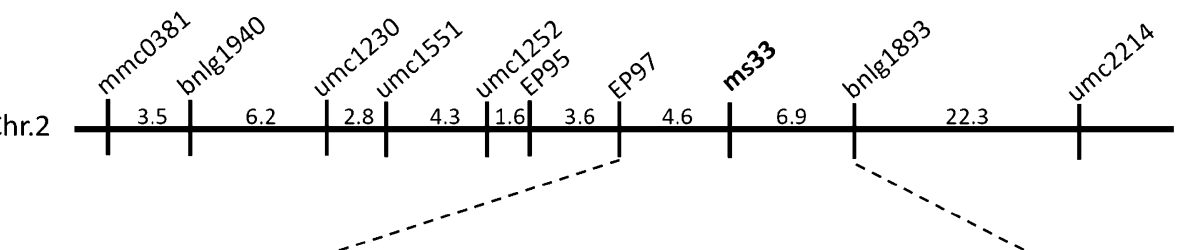

Marker

Genetic map (cM)

$\mathrm{n}=228$

b

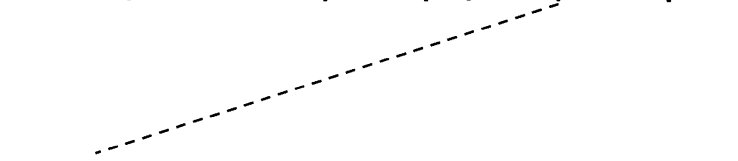

b

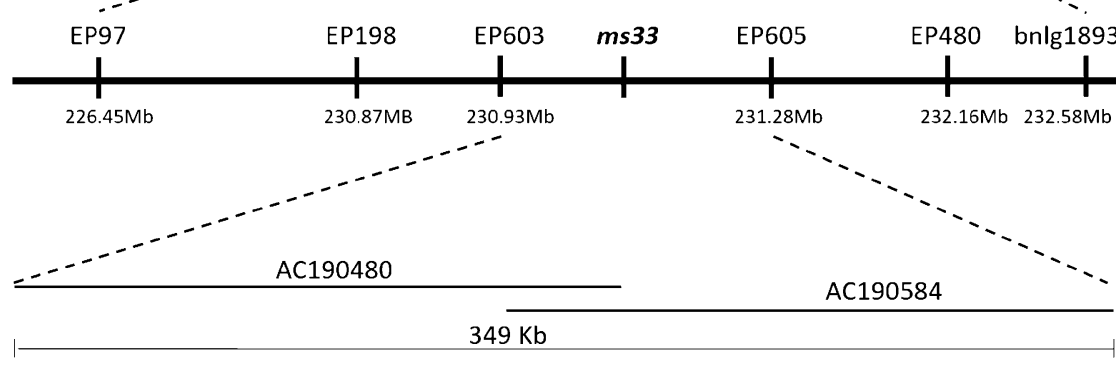

Marker

Physical map (Mb)

$\mathrm{n}=463$

C

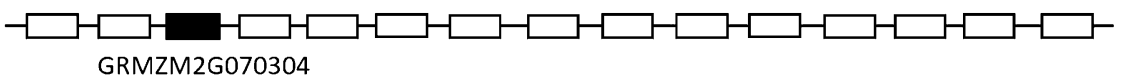

Gene models

Fig. 3 Map-based cloning of the maize $m s 33$ gene. a Primary mapping of the $m s 33$ gene between markers EP97 and bnlg1893. b Fine mapping of the $m s 33$ gene to an interval of nearly $349 \mathrm{~kb}$ between markers EP603 and EP605. c The 15 putative gene models in the interval. Among them, GRMZM2G070304, similar to the rice OsGPAT3 gene, is the candidate gene. $n$ the number of the male-sterile plants used in the $\mathrm{F}_{2}$ mapping population is showed on the right 

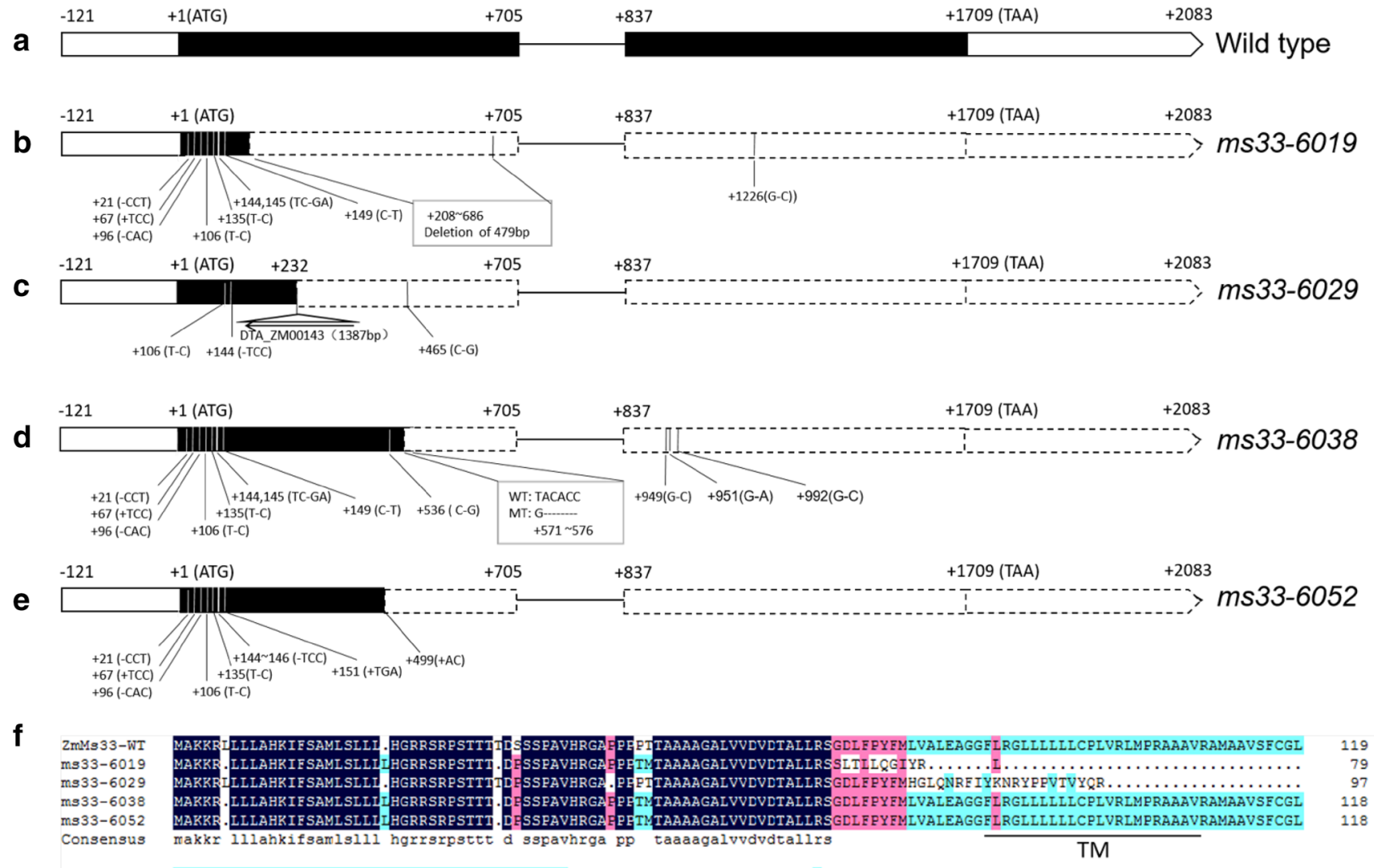

ma33-6029

ms $33-6029$

ms33-6052

Consensis

RAMRERAGRAVI

Fig. 4 The gene structure and predicted amino acid sequences of the maize ZmMs33 candidate gene (GRMZM2G070304). a A schematic representation of two exons and one intron of $\mathrm{ZmMs} 33$ in wild type. +1 indicates the putative starting nucleotide of translation, and the stop codon (TAA) is +1709 in wild type. Black boxes indicate exons, and intervening lines indicate introns. b In ms33-6019, besides the 479-bp deletion at the +208-686, there are three InDels and five SNPs in Exon 1 and one SNP in Exon 2. $\mathbf{c}$ In $m s 33-6029$, besides the transposon insertion at the +232 site, there are two SNPs and a 3-bp deletion. d In $m s 33-6038$, besides the 5-bp deletion at the $+572-576$, there are three InDels and seven SNPs in Exon 1 and three SNPs in Exon 2. e In $m s 33-6052$, besides the 2-bp insertion at the +499-500, there are five InDels and two SNPs in Exon 1. $\mathbf{f}$ The insertion or deletion mutations in $m s 33-6019, m s 33-6029, m s 33-6038$ and $m s 33-6052$ caused frame-shift mutations and altered the reading frame after the 69th, 77th, 188th and 160th amino acid, respectively, resulting in a lack of the conserved acyltransferase domain (AT), which contains four conserved motifs. The putative transmembrane domain (TM) and the conserved AT domain are underlined 
and $m s 33-6052$ mutants (Fig. 4d, e), which also resulted in deletion of two amino acids, one amino-acid insertion, and four amino-acid changes (Fig. 4f).

By using Agrobacterium-mediated maize transformation, 15 transgenic lines with the coding sequence of GRMZM2G070304 driven by its own promoter $(1.8 \mathrm{~kb})$ restored male fertility in maize ms33-6029 mutant (Fig. 5; Table S4). Furthermore, the GRMZM2G070304 gene was further confirmed as the $m s 33$ gene by targeted knockouts using a CRISPR/Cas9 system (Figs. 6, 7). The maize Hi-II hybrid line was transformed with three vectors (1 single gRNA/Cas9 vector and 2 double gRNA/Cas9 vectors; Fig. 6) targeting different sites of endogenous $Z m M s 33$. Site-directed mutations were observed at all targeted sites by DNA sequencing analysis in more than 30 independent transgenic events (Fig. S8 and Table S5). The three types of $\mathrm{T}_{0}$-generation maize plants homozygous for null alleles of ZmMs33 were observed to be frame-shift mutations by 1-, 192- and 453-bp deletions (Fig. 7). These results showed that GRMZM2G070304 is responsible for restoring the male-sterile phenotype in the ms33 mutants; hereafter, we refer to GRMZM2G070304 as ZmMs33.

\section{$\mathrm{ZmMs33}$ is mainly expressed in the anther and root tissues}

To analyse the expression pattern of ZmMs33, semiquantitative RT-PCR was performed. The results revealed that $Z m M s 33$ was mainly expressed in premature anthers from the quartet to early-vacuolate microspore stages and in root tissues at the fifth leaf growth stage (Fig. 8a, b). Compared to the wild type, the expression of ZmMs 33 was hardly detected in the premature anthers of the $m s 33-6029$ mutant (Fig. 8c). The anther-preferential expression pattern of $Z m M s 33$ is consistent with its function in anther development. Although relatively higher levels of ZmMs33 expression were also detected in root tissues during the fifth leaf growth stage, and further confirmed to be ZmMs 33 cDNA by sequencing the RT-PCR products (Fig. S4), no obvious morphological abnormalities were observed in the roots of
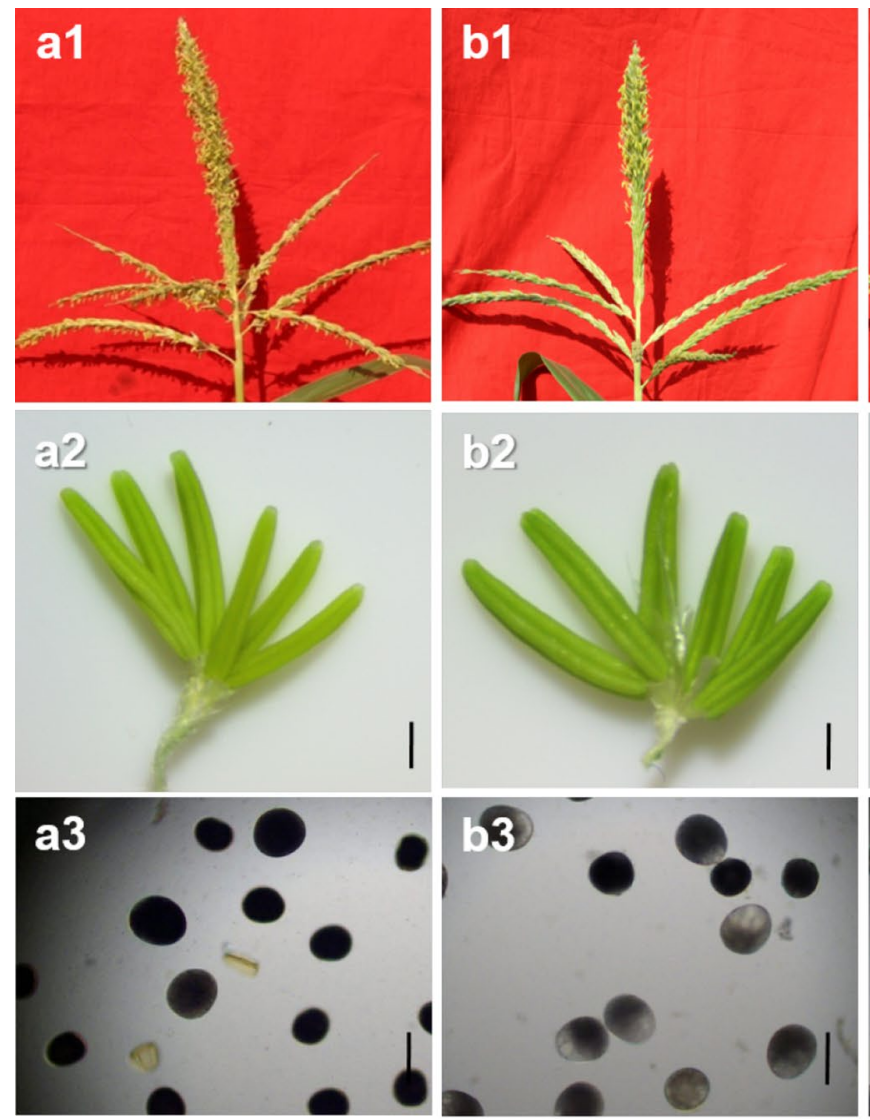

WT (Ms33-6029)

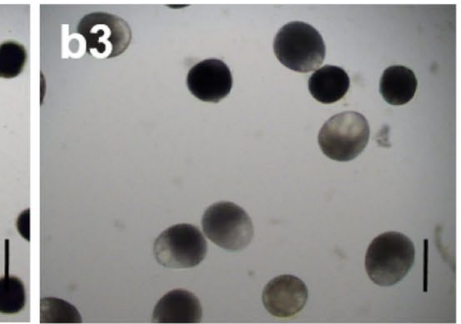

C-ms33-6029-1

Fig. 5 Functional complementation of the maize ms33-6029 mutant. a1-d1 Phenotypes of tassels, a2-d2 spikelets and a3-d3 pollen grains stained with $\mathrm{I}_{2}-\mathrm{KI}$ in a1-a3 the wild type, d1-d3 $m s 33-6029$ mutant and two complemented transgenic lines b1-b3 C-ms33-
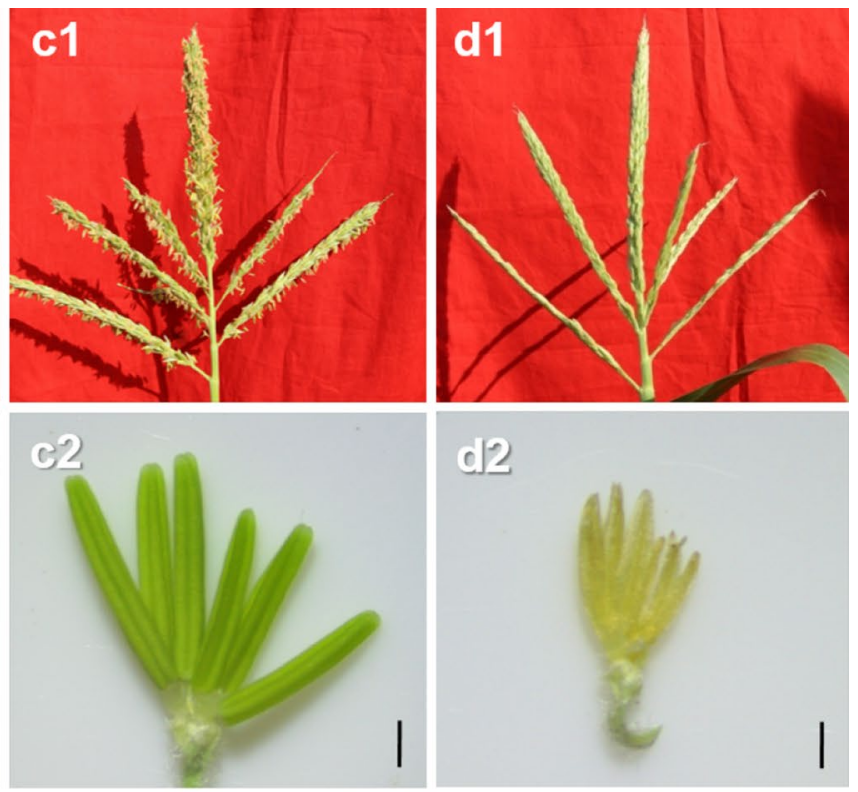

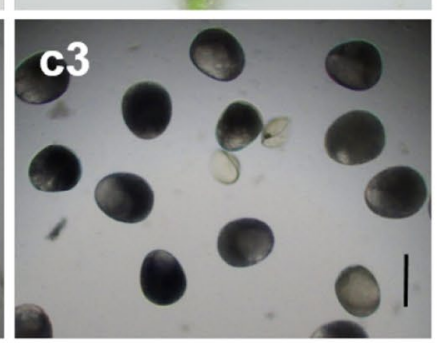

C-ms33-6029-2

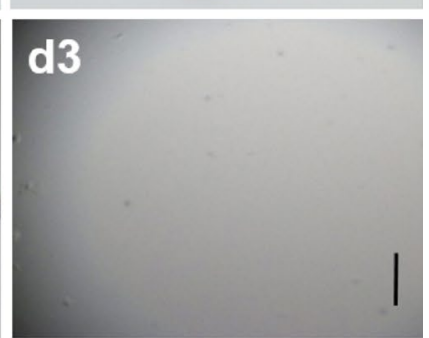

$m s 33-6029$
6029-1 (pMs33::Ms33/ms33-6029) and c1-c3 C-ms33-6029-2 (pMs33::Ms33/ms33-6029), respectively. Bars = $1 \mathrm{~mm}(\mathbf{a 2}-\mathbf{d 2})$, $100 \mu \mathrm{m}(\mathbf{a 3}-\mathbf{d} 3)$ 
Fig. 6 Physical maps of three CRISPR/Cas9 vectors carrying a one-gRNA, b, $\mathbf{c}$ two-gRNAs and their corresponding target sites in the ZmMs33 gene
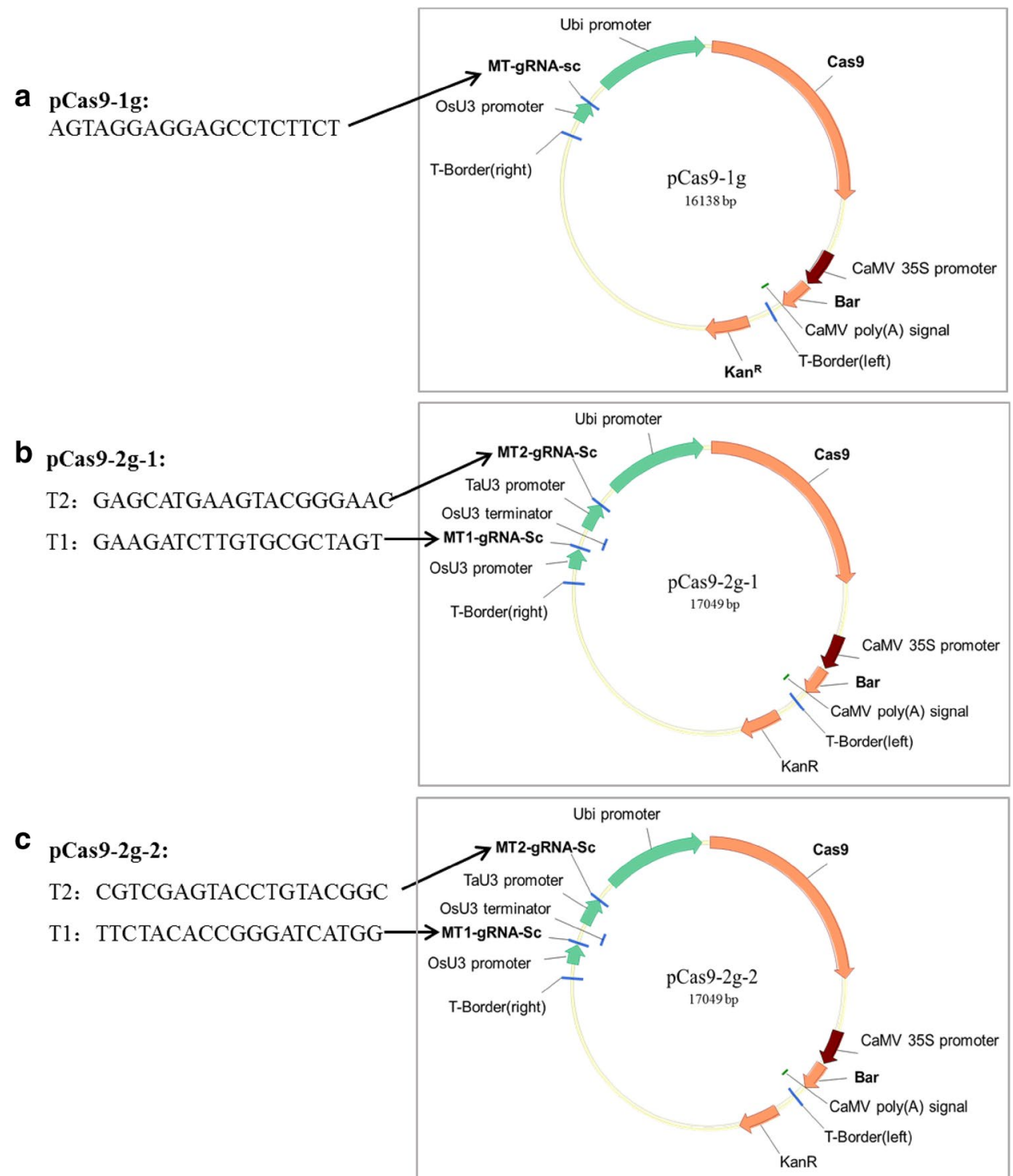

ms33, suggesting a possible redundant function of ZmMs33related homologues in the root tissues.

\section{ZmMs33 is the orthologue of OsGPAT3, belonging to a monocot-specific sn-2 GPAT family}

To elucidate the evolutionary relationship between $\mathrm{ZmMs} 33$ and its close homologues, the ZmMs33 protein sequence was used in BLAST $\mathrm{P}_{\mathrm{p}}$ queries in NCBI, MaizeGDB and RGAP databases. Meanwhile, we used AtATS1 and AtGPAT9 protein sequences to search for the putative $s n-1$ GPATs in maize and rice. Consequently, there are 18 and 20 putative GPATs in the maize and rice genomes, respectively (Table S6). Because the GPAT family is relatively large, we only collected a total of 60 protein sequences, including all GPAT members from Arabidopsis, maize and rice, and the top 12 most closely related ZmMs33 homologues from other 12 plant species. We constructed a neighbourjoining phylogenetic tree of the 60 GPAT protein sequences, which are grouped into two main clades (Fig. 9). The $s n-1$ clade included eight members that could be divided into two subclades: the plastidic AtATS1-related subclade and the ER-bound AtGPAT9-related subclade. The $s n-2$ GPAT clade included all the other 52 GPAT members and was classified into two subclades. The first subclade was divided into three groups: the AtGPAT4/6/8-related group required for the biosynthesis of cutin, the AtGPAT5/7-related group associated with the biosynthesis of suberin, and the AtGPAT1-related group. The second subclade was related to AtGPAT2/3, which have been less functionally characterized. ZmMs33 was located to the monocot branch including OsGPAT3, a rice GPAT (LOC_Os12g37600), a maize GPAT (GRMZM2G033767) and eight other homologues in monocots such as Oryza brachyantha $(\mathrm{Ob})$, Sorghum 

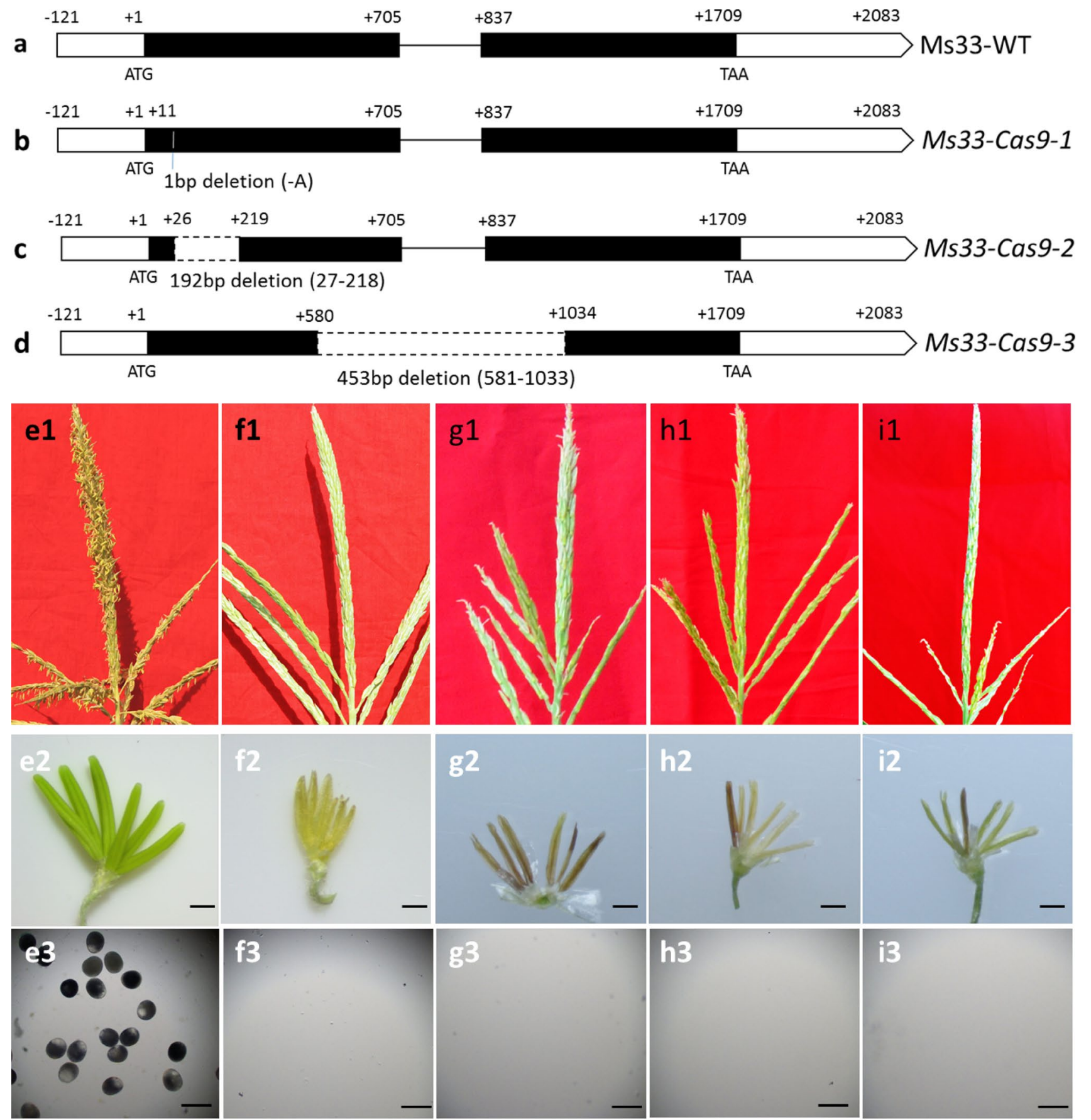

WT (Ms33-6029)

$m s 33-6029$

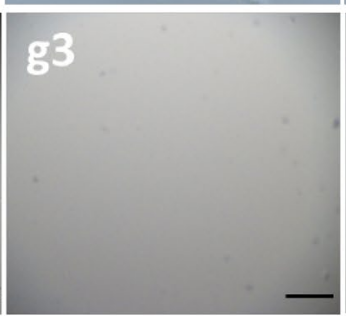

Ms33-Cas9-1

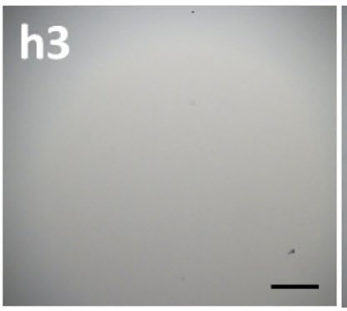

Ms33-Cas9-2

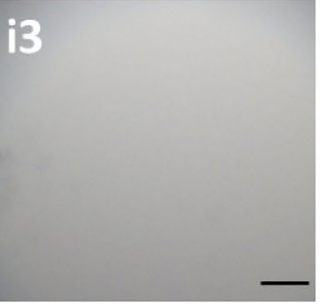

Ms33-Cas9-3

Fig. 7 Phenotypes of tassels, spikelets and pollen grains in three knockout lines generated by a CRISPR/Cas9 system. Compared with the wild-type (a) sequence, deletions of 1, 192, and 453 bp were detected in the three knockout lines Ms33-Cas9-1 (b), Ms33-Cas9-2 (c), and Ms33-Cas9-3 (d), respectively. e1 Wild-type tassel, e2 spike-

bicolor (Sb), Triticum aestivum (Ta), Bradchypodium distachyon (Bd), Aegilops taushii (Ats), Dichanthelium oligosanthes (Do), Hordeum vulgare (Hv) and Setaria italica (Si), whereas Arabidopsis AtGPAT2/3 occupied a relatively let and $\mathbf{e} 3$ mature pollen grains stained with $1 \%$ I2-KI solution. f2 The ms33-6029 mutant anthers were not exserted from f1 the tassel, and $\mathbf{f 3}$ no pollen grains were present in the anthers. g1-i1 The tassels, g2-i2 spikelets and $\mathbf{g 3}-\mathbf{i} 3$ absence of pollen grains in the three knockout lines (Ms33-Cas9-1 3). Bars = 1 mm (e2-i2), $150 \mu \mathrm{m}(\mathbf{e} 3-\mathbf{i} 3)$

distant dicot branch with four homologues in dicots such as Glycine max (Gm), Brassica napus (Bn), Raphanus sativus (Rs) and Brassica rapa (Br) (Fig. 9). Based on the predicted amino acid sequence alignment, ZmMs33 shares 


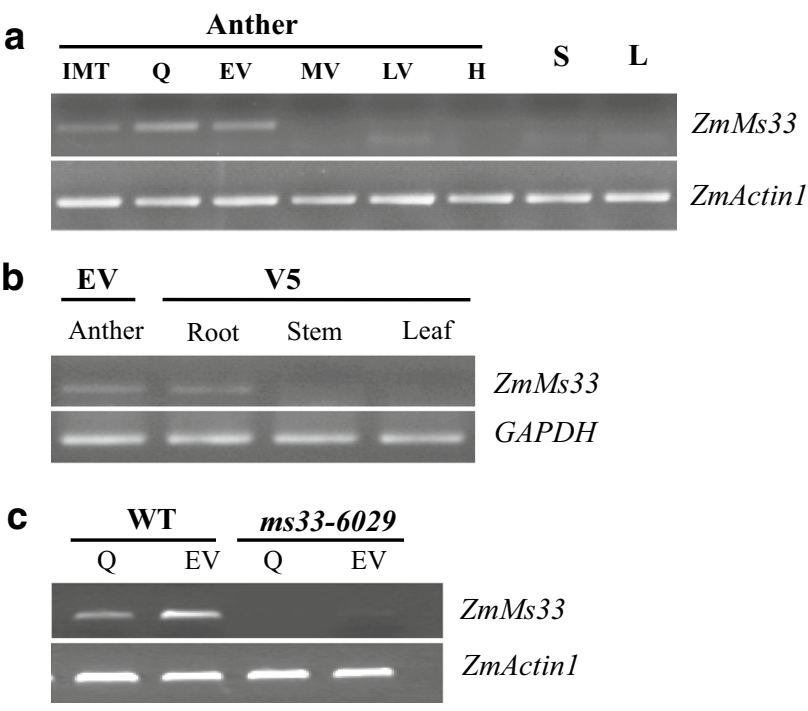

Fig. 8 Expression patterns of the maize ZmMs33 gene by RT-PCR analysis. a Maximum expression was observed in the anther during the meiosis quartet to early vacuolate microspore stages. b ZmMs33 expression in maize root at the fifth leaf growth stage, but no expression was detected in maize stem and leaf. c Comparison of ZmMs33 expression in the anther of wild type and the ms33-6029 mutant at the meiosis quartet and early vacuolate microspore stages. ZmMs33 expression data were normalized against ZmActin1 or GAPDH. Different anther developmental stages are shown: IMT immature tassels, $Q$ meiosis quartets, $E V$ early vacuolate microspore, $M V$ middle vacuolate microspore, $L V$ late vacuolate microspore, $H$ heading, $S$ stem, $L$ leaf, $V 5$ the fifth leaf growth stage

$74,69,80$ and $79 \%$ similarity with the orthologues of barley (AK376456), rice OsGPAT3 (Os11g45400), sorghum (XM_002449936), and millet (XM_004979921), respectively. Moreover, all the orthologues have a conserved AT domain at the same place (Fig. 10). Based on a BLAST $P$ search of the NCBI database, ZmMs33 was relatively close to OsGPAT3 (Table S3). Therefore, ZmMs33 is the orthologue of rice OsGPAT3, which may represent a unique $s n-2$ GPAT specific to monocots.

\section{Discussion}

\section{ms33 is a complete male-sterile mutant and desirable for improving maize male-sterile line in hybrid seed production}

In this study, we confirmed that $m s 33$ is a complete malesterile mutant with no exerted anthers and no mature pollen grains (Figs. 1 and S2). Loss of ZmMs33 expression in anthers (Fig. 8c) disrupts tapetum development and metabolism, which results in defective anther cuticle, blocked pollen exine formation, and eventually complete abortion of pollen grains (Figs. 2 and S3). The genetic analysis of the two $\mathrm{F}_{2}$ population derived from the crosses of $m s 33$ $6029 \times$ Chang 7-2 and $m s 33-6038 \times$ Chang7-2 showed the 3:1 segregation ratio of the male-fertile to male-sterile plants, respectively (Table 1), which suggested both $m s 33-6029$ and ms33-6038 were single recessive mutants. As the complete male-sterile phenotype of $m s 33$ mutants was genetically stable under different environments (Patterson 1995; Trimnell et al. 1999), ms33 is a desirable genetic resource for improving maize male-sterile lines in hybrid seed production.

\section{Confirming gene function of $\mathrm{ZmMs} 33$ based on targeted mutagenesis induced by a CRISPR/Cas9 system}

ms33 was narrowed down to an interval of $349 \mathrm{~kb}$ on chromosome $2 \mathrm{~L}$, and there were 15 putative genes in the interval (Fig. 3 and Table S3). To verify that GRMZM2G070304 is the ZmMs33 gene, two experiments were carried out: (1) functional complementation and (2) site-targeted mutagenesis using a CRISPR/Cas9 system. Transgenic lines with the coding sequence of GRMZM2G070304 driven by its native promoter restored male fertility (Fig. 5). Based on targeted knockouts induced by the CRISPR/Cas9 system (Fig. 6), all the three types of knockout lines with different deletions in the GRMZM2G070304 coding sequence showed a complete male-sterile phenotype (Fig. 7). These results demonstrate that GRMZM2G070304 is responsible for restoring the male-sterile phenotype of the ms 33 mutants.

Recently, the CRISPR/Cas9 system has been widely used in gene function confirmation and genetic improvement in maize and other crops. A public-sector system (ISU Maize CRISPR) utilizing Agrobacterium-delivered CRISPR/Cas9 was developed for high-frequency targeted mutagenesis in four maize genes: ZmAgo18a, ZmAgo18b, al and a4 (Char et al. 2017). The CRISPR/Cas9 system was also successfully used for functional confirmation of IPEI, APVI and ARGOS8 genes in maize (Chen et al. 2017; Shi et al. 2017; Somaratne et al. 2017). Furthermore, this system has been used in other crops such as rice ( $\mathrm{Li}$ et al. 2016; Woo et al. 2015), wheat (Zhang et al. 2016), cotton (Wang et al. 2018), soybean (Cai et al. 2018) and Camelina sativa (Jiang et al. 2017).

In this study, the maize Hi-II hybrid line was transformed with three CRISPR/Cas9 vectors (Fig. 6) targeting different sites of endogenous ZmMs33. The three types of $\mathrm{T}_{0}$-generation maize plants homozygous for null alleles of ZmMs33 were observed to be frame-shift mutations by 1-, 192- and 453-bp deletions (Fig. 7). All these site-targeted null mutant lines showed a completely male-sterile phenotype, indicating that both single gRNA/Cas9 and double gRNA/Cas9 constructs are highly efficient in producing sitespecific gene mutants of ZmMs33. Therefore, it is feasible 
Fig. 9 Phylogenetic analysis of ZmMs33 and related proteins. The evolutionary analyses were conducted in MEGA7 using the maximum likelihood method based on the Poisson correction model. The tree with the greatest log likelihood is shown. The analyses involved 48 amino acid sequences from Zea mays $(\mathrm{Zm})$, Oryza sativa $(\mathrm{Os})$ and Arabidopsis thaliana (At), and 12 putative GPATs from other plants, including Oryza brachyantha $(\mathrm{Ob})$, Sorghum bicolor (Sb), Triticum aestivum (Ta), Bradchypodium distachyon (Bd), Aegilops tauschii (Ats), Dichanthelium oligosanthes (Do), Hordeum vulgare (Hv), Setaria italica (Si), Glycine max (Gm), Brassica napus (Bn), Raphanus sativus (Rs) and Brassica rapa $(\mathrm{Br})$

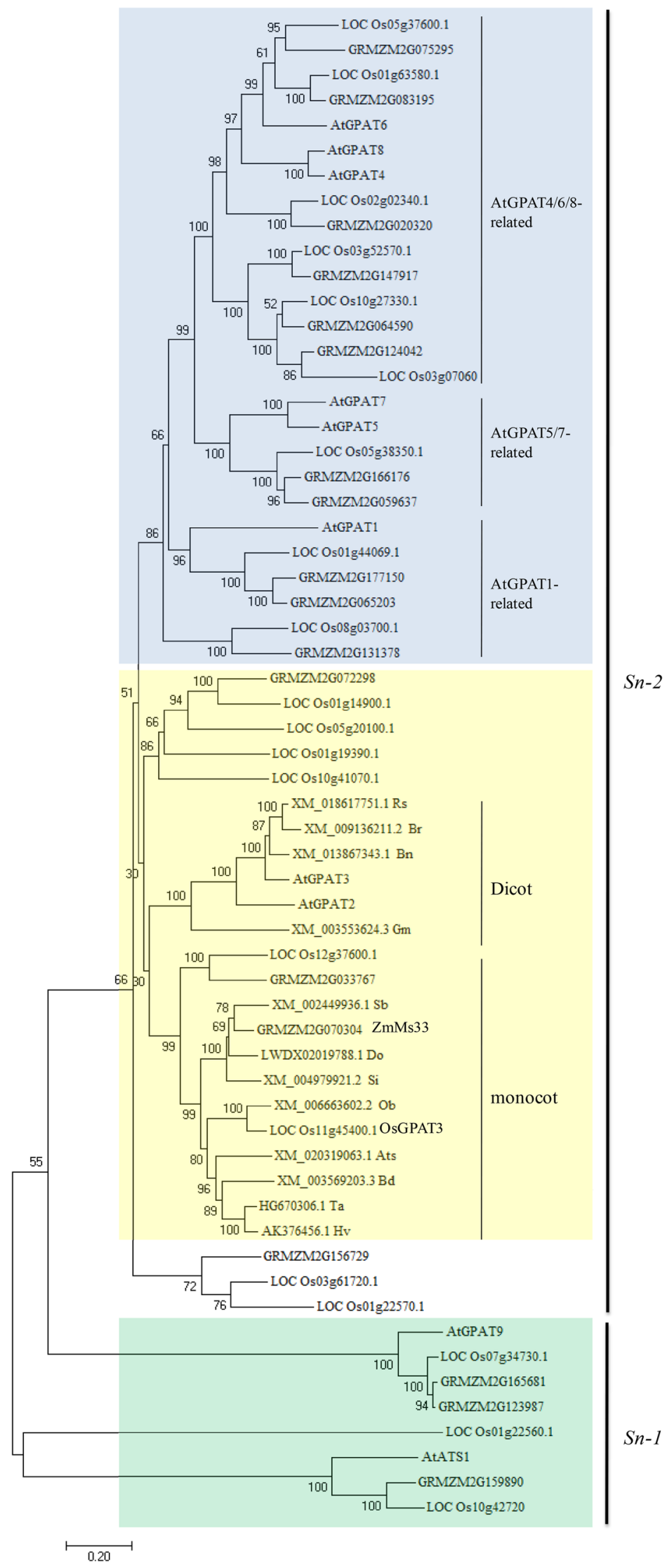




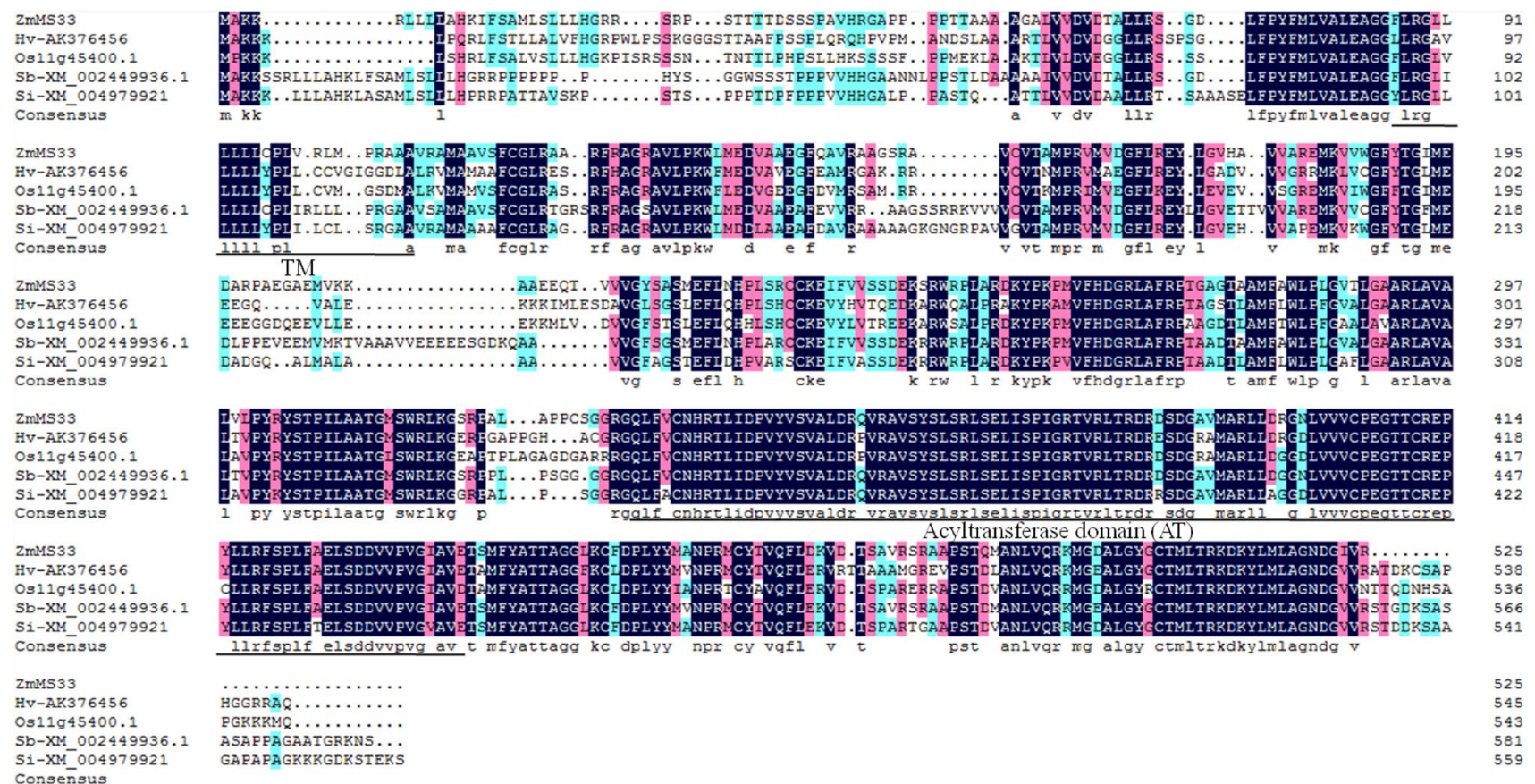

Fig. 10 The amino acid sequence alignment of maize ZmMs33 and orthologous proteins. Hv-AK376456 (Hordeum vulgare), Os11g45400.1 (OsGPAT3, Oryza sativa), Sb-XM_002449936.1

and efficient to use the CRISPR/Cas9 system for gene functional confirmation in maize.

\section{ZmMs33 and OsGPAT3 represent a monocot-specific sn-2 GPAT family}

ZmMs33 encodes a GPAT protein with a conserved AT domain and shares high similarity with rice OsGPAT3 (Men et al. 2017). Maize $m s 33$ and rice osgpat3 mutants showed the similar male-sterile phenotypes, anther and pollen defects (Figs. 1, 2, S2 and S3). Moreover, similar with expression patterns of the rice OsGPAT3 gene, ZmMs33 was also expressed preferentially in maize immature anthers and root tissues (Fig. 8). Therefore, ZmMs33 appears to be an orthologue of OsGPAT3 and is required for male fertility in maize.

The land plant GPATs belong to a $s n-2$ GPAT family that differs from animal GPATs (Yang et al. 2012). The orthologues of ZmMs33 were found in 14 flowering plants, including 9 monocots and 5 dicots, and ZmMs33 and OsGPAT3 belong to a monocot-specific $s n$-2 GPAT family, similar to the uncharacterized AtGPAT2 and AtGPAT3 in Arabidopsis (Fig. 9, Table S6). Although no enzyme activity was detected for AtGPAT2 and AtGPAT3, and their mutants showed no obvious phenotype in plant development (Yang et al. 2012), the ms33 and osgpat 3 mutants displayed similar defective phenotypes in anther and complete male sterility
(Sorghum bicolor), Si-XM_004979921 (Setaria italica). The putative transmembrane (TM) domain and conserved acyltransferase (AT) domain are underlined

(Men et al. 2017). We propose that ZmMs33 and OsGPAT3, together with their homologues in monocots, may have evolved divergently, leading to diversified functions different from those in dicots. Mutations in maize ZmMs33 and rice OsGPAT3 led to the same complete male-sterile phenotype, demonstrating that this kind of monocot-specific $s n-2$ GPAT protein may be functionally conserved in monocot plants, where they play an indispensable role in male reproduction that is distinct from the role of their dicot counterparts. Therefore, these monocot-specific $s n-2$ GPAT genes may have potential application value in hybrid seed production in monocots.

\section{ZmMs33 is the first GPAT gene identified in maize, and it controls male fertility through mediating glycerolipid synthesis}

The tapetal lipid metabolism contributes significantly to anther wall cuticle and pollen exine formation (Shi et al. 2015). Glycerol is an essential backbone of plant polyesters such as suberin; however, its presence in plant cutin was discovered only comparatively recently (Graca et al. 2002; Pollard et al. 2008). GPATs play vital roles in mediating the initial step of glycerolipid synthesis pathway (Beisson et al. 2012; Yang et al. 2012).

In Arabidopsis, AtGPAT1 is important for male fertility, and the atgpat1 mutant exhibits a reduced ER stacks and 
mitochondrial dysfunction in the tapetum, thus leading to delayed programmed cell death of the tapetum cell layer and male semi-sterility. AtGPAT6 has a similar expression pattern to AtGPATl in anther development. In the atgpat6 mutant, the ER system in the tapetum was severely impaired, ultimately resulting in defective pollen wall formation and male semi-sterility. The atgpat1atgpat6 double mutant showed a defect of microspore release from tetrads and complete male sterility (Li et al. 2012; Zheng et al. 2003).

To date, there has been little gene isolation and characterization of GPATs in monocots. More recently, one member of the rice GPAT family, OsGPAT3, was found to be involved in the anther development and pollen formation in rice (Men et al. 2017). However, none of the GPAT genes in maize has been investigated until now. ZmMs33 is the first GPAT gene identified in maize. Loss of ZmMs33 expression in anthers disrupts anther development, leading to eventually complete abortion of pollen grains (Figs. 1, 2, S2, S3 and 8). Together with our data on $\mathrm{ZmMs} 33$ and the functions of OsGPAT3, AtGPAT1 and AtGPAT6, we concluded that these GPATs play important roles in anther development and male fertility through mediating glycerolipid synthesis in plants.

\section{The potential application of the ZmMs33 gene in maize male sterile-line breeding and hybrid seed production}

To date, hundreds of recessive genetic male-sterile mutants have been identified in plants, but their application in plant breeding and hybrid seed production has been limited because of the inability to propagate a pure male-sterile line via self-pollination. Recently, several strategies for maintaining and propagating male-sterile lines have been proposed based on the transgenic lines in plants such as maize (Wu et al. 2016; Zhang et al. 2018), rice (Chang et al. 2016), tobacco (Millwood et al. 2016) and Arabidopsis (Gils et al. 2008).

Most recently, to decrease the transgene transmission rate of the transgenic maintainer line through pollen, we developed a Multi-Control Sterility (MCS) system by transforming MCS constructs into the $m s 7$ mutant (Zhang et al. 2018). The MCS constructs contained five functional modules: (1) a male fertility gene $Z m M s 7$, (2) two pollendisrupted genes (ZmAA and Dam), (3) a screenable fluorescent colour marker gene (DsRed2 or mCherry) and (4) an herbicide-resistant gene (Bar). The MCS constructs harbour five functional modules with the addition of $\mathrm{Bar}$ and Dam genes, which can ensure high purity of the malesterile parent line by appropriate herbicide spraying of seedlings, and greatly decrease the transgene transmission rate as well as transgene flow risk through the use of two pollen-disrupting modules (Zhang et al. 2018). Therefore, the MCS system could be reconstructed based on the novel male-fertile ZmMs33 gene and its mutants ( $m s 33-6029$ and $m s 33-6038$ ), and it is feasible to breed $m s 33$ male-sterile lines by combining traditional backcrossing and marker assisted selection (MAS) methods.

The orthologues of $\mathrm{ZmMs} 33$ were found in 14 flowering plants, and the GPAT family is divergent and has many members in major crops, including sorghum, barley and millet (Fig. 9), which have flowers that are not amenable to manual emasculation. Furthermore, ZmMs33, OsGPAT3 and their orthologues were found to be conserved in monocot plants and required for plant male gametogenesis (Fig. 10). Therefore, a reverse genetic approach could be adopted using targeted mutagenesis technologies like the CRISPR/Cas9 system (Kamthan et al. 2016; Zhang et al. 2016) and programmable DNA endonucleases (Cigan et al. 2017) or using chimeric repressor gene-silencing technology (Mitsuda et al. 2006) and RNAi (Fernández Gómez and Wilson 2014), which would lead to specific mutations in the corresponding orthologues of ZmMs33 and produce male-sterile mutants in these crops. This will greatly enhance our understanding of the molecular mechanism of GPATs that are essential for male fertility in plants. In addition, the MCS system could be transferred into other major crops using artificial $m s$ mutants and the corresponding fertility restoration genes, which will greatly expand the potential to produce male-sterile lines and hybrid seeds of important crops.

Acknowledgements This work was supported by the National Key R\&D Program of China (2017YFD0102001, 2017YFD0101201), the National Natural Science Foundation of China (31771875), the Fundamental Research Funds for the Central Universities of China (06500060, FRF-BR-17-009A, FRF-BR-17-010A, FRF-BR17-011A), the International S\&T Cooperation Program of China (2015DFA30640), the National Key Technology R\&D Program of China (2014BAD01B02), and the Beijing Science \& Technology Plan Program (Z161100000916013). We thank Yanbao Tian (Institute of Genetics and Developmental Biology Chinese Academy of Sciences) for help in SEM sample preparation and observation.

Author contribution statement $\mathrm{KX}$ constructed the plasmids and performed the functional complementation; SW performed part of the experiments, and drafted the manuscript; ZL performed the bioinformatic analysis; YZ performed the gene mapping; DZ performed the phenotypic observation, and gene expression; $\mathrm{ZD}$ and $\mathrm{XA}$ performed the SEM analysis of anthers; TZ and SZ provided technical assistance to $\mathrm{KX}$; SL and JL provided assistance to SW; XW designed and supervised the experiments, and revised the manuscript. All authors read and approved the final manuscript.

\section{Compliance with ethical standards}

Conflict of interest The authors declare that they have no conflict of interest.

Ethical approval The authors declare that the experiments comply with the current laws of China. 
Open Access This article is distributed under the terms of the Creative Commons Attribution 4.0 International License (http://creativeco mmons.org/licenses/by/4.0/), which permits unrestricted use, distribution, and reproduction in any medium, provided you give appropriate credit to the original author(s) and the source, provide a link to the Creative Commons license, and indicate if changes were made.

\section{References}

Albertsen MC, Phillips RL (1981) Developmental cytology of 13 genetic male sterile loci in maize. Can J Genet Cytol 23:195-208

Albertsen MC, Fox T, Trimnell M, Wu Y, Lowe L, Li B, Faller M (2009) Mscal nucleotide sequences impacting plant male fertility and method of using same. US patent US20090038027A1

Albertsen M, Fox T, Leonard A, Li B, Loveland B, Trimnell M (2016) Cloning and use of the $m s 9$ gene from maize. US patent US20160024520A1

Beisson F, Li-Beisson Y, Pollard M (2012) Solving the puzzles of cutin and suberin polymer biosynthesis. Curr Opin Plant Biol 15:329-337

Cai Y, Chen L, Liu X, Guo C, Sun S, Wu C, Jiang B, Han T, Hou W (2018) CRISPR/Cas9-mediated targeted mutagenesis of GmFT2a delays flowering time in soya bean. Plant Biotechnol J 16:176-185

Chang Z, Chen Z, Wang N, Xie G, Lu J, Yan W, Zhou J, Tang X, Deng XW (2016) Construction of a male sterility system for hybrid rice breeding and seed production using a nuclear male sterility gene. Proc Natl Acad Sci 113:14145-14150

Char SN, Neelakandan AK, Nahampun H, Frame B, Main M, Spalding $\mathrm{MH}$, Becraft PW, Meyers BC, Walbot V, Wang $\mathrm{K}$, Yang B (2017) An Agrobacterium-delivered CRISPR/Cas9 system for high-frequency targeted mutagenesis in maize. Plant Biotechnol J 15:257-268

Chen S, Songkumarn P, Liu J, Wang GL (2009) A versatile zero background $\mathrm{T}$-vector system for gene cloning and functional genomics. Plant Physiol 150:1111-1121

Chen X, Zhang H, Sun H, Luo H, Zhao L, Dong Z, Yan S, Zhao C, Liu R, Xu C, Li S, Chen H, Jin W (2017) IRREGULAR POLLEN EXINE1 Is a novel factor in anther cuticle and pollen exine formation. Plant Physiol 173:307-325

Cigan AM, Unger E, Xu RJ, Kendall T, Fox TW (2001) Phenotypic complementation of $m s 45$ maize requires tapetal expression of MS45. Sex Plant Reprod 14:135-142

Cigan AM, Singh M, Benn G, Feigenbutz L, Kumar M, Cho MJ, Svitashev S, Young J (2017) Targeted mutagenesis of a conserved anther-expressed P450 gene confers male sterility in monocots. Plant Biotechnol J 15:379-389

Cui X, Wise RP, Schnable PS (1996) The rf2 nuclear restorer gene of male-sterile T-cytoplasm maize. Science 272:1334-1336

Djukanovic V, Smith J, Lowe K, Yang M, Gao H, Jones S, Nicholson MG, West A, Lape J, Bidney D, Carl Falco S, Jantz D, Alexander Lyznik L (2013) Male-sterile maize plants produced by targeted mutagenesis of the cytochrome P450-like gene (MS26) using a re-designed I-CreI homing endonuclease. Plant J 76:888-899

Eyster LA (1921) Heritable characters of maize. VII. Male sterile. J Hered 12:138-141

Feng Y, Zheng Q, Song H, Wang Y, Wang H, Jiang L, Yan J, Zheng Y, Yue B (2015) Multiple loci not only Rf3 involved in the restoration ability of pollen fertility, anther exsertion and pollen shedding to $\mathrm{S}$ type cytoplasmic male sterile in maize. Theor Appl Genet 128:2341-2350
Fernández Gómez J, Wilson ZA (2014) A barley PHD finger transcription factor that confers male sterility by affecting tapetal development. Plant Biotechnol J 12:765-777

Fox T, DeBruin J, Haug Collet K, Trimnell M, Clapp J, Leonard A, Li B, Scolaro E, Collinson S, Glassman K, Miller M, Schussler J, Dolan D, Liu L, Gho C, Albertsen M, Loussaert D, Shen B (2017) A single point mutation in Ms44 results in dominant male sterility and improves nitrogen use efficiency in maize. Plant Biotechnol J 15:942-952

Gils M, Marillonnet S, Werner S, Grutzner R, Giritch A, Engler C, Schachschneider R, Klimyuk V, Gleba Y (2008) A novel hybrid seed system for plants. Plant Biotechnol J 6:226-235

Gomez JF, Talle B, Wilson ZA (2015) Anther and pollen development: a conserved developmental pathway. J Integr Plant Biol 57:876-891

Graca J, Schreiber L, Rodrigues J, Pereira H (2002) Glycerol and glyceryl esters of omega-hydroxyacids in cutins. Phytochemistry 61:205-215

Hu YM, Tang JH, Yang H, Xie HL, Lu XM, Niu JH, Chen WC (2006) Identification and mapping of Rf-I an inhibitor of the Rf5 restorer gene for Cms-C in maize (Zea mays L.). Theor Appl Genet 113:357-360

Jiang WZ, Henry IM, Lynagh PG, Comai L, Cahoon EB, Weeks DP (2017) Significant enhancement of fatty acid composition in seeds of the allohexaploid, Camelina sativa, using CRISPR/Cas9 gene editing. Plant Biotechnol J 15:648-657

Jung KH, Han MJ, Lee YS, Kim YW, Hwang I, Kim MJ, Kim YK, Nahm BH, An G (2005) Rice Undeveloped Tapetum1 is a major regulator of early tapetum development. Plant Cell 17:2705-2722

Kamthan A, Chaudhuri A, Kamthan M, Datta A (2016) Genetically modified (GM) crops: milestones and new advances in crop improvement. Theor Appl Genet 129:1639-1655

Kelliher T, Walbot V (2012) Hypoxia triggers meiotic fate acquisition in maize. Science 337:345-348

Kohls S, Stamp P, Knaak C, Messmer R (2011) QTL involved in the partial restoration of male fertility of C-type cytoplasmic male sterility in maize. Theor Appl Genet 123:327-338

Kumar S, Stecher G, Tamura K (2016) MEGA7: molecular evolutionary genetics analysis version 7.0 for bigger datasets. Mol Biol Evol 33:1870-1874

Li Y, Beisson F, Koo AJ, Molina I, Pollard M, Ohlrogge J (2007) Identification of acyltransferases required for cutin biosynthesis and production of cutin with suberin-like monomers. Proc Natl Acad Sci USA 104:18339-18344

Li XC, Zhu J, Yang J, Zhang GR, Xing WF, Zhang S, Yang ZN (2012) Glycerol-3-phosphate acyltransferase 6 (GPAT6) is important for tapetum development in Arabidopsis and plays multiple roles in plant fertility. Mol Plant 5:131-142

Li J, Meng X, Zong Y, Chen K, Zhang H, Liu J, Li J, Gao C (2016) Gene replacements and insertions in rice by intron targeting using CRISPR-Cas9. Nat Plants 2:16139

Ma J, Skibbe DS, Fernandes J, Walbot V (2008) Male reproductive development: gene expression profiling of maize anther and pollen ontogeny. Genome Biol 9:R181

Men X, Shi J, Liang W, Zhang Q, Lian G, Quan S, Zhu L, Luo Z, Chen M, Zhang D (2017) Glycerol-3-Phosphate Acyltransferase 3 (OsGPAT3) is required for anther development and male fertility in rice. J Exp Bot 68:513-526

Millwood RJ, Moon HS, Poovaiah CR, Muthukumar B, Rice JH, Abercrombie JM, Abercrombie LL, Green WD, Stewart CN Jr (2016) Engineered selective plant male sterility through pollen-specific expression of the EcoRI restriction endonuclease. Plant Biotechnol J 14:1281-1290

Mitsuda N, Hiratsu K, Todaka D, Nakashima K, Yamaguchi-Shinozaki K, Ohme-Takagi M (2006) Efficient production of male and 
female sterile plants by expression of a chimeric repressor in Arabidopsis and rice. Plant Biotechnol J 4:325-332

Moon J, Skibbe D, Timofejeva L, Wang CJ, Kelliher T, Kremling K, Walbot V, Cande WZ (2013) Regulation of cell divisions and differentiation by MALE STERILITY32 is required for anther development in maize. Plant J 76:592-602

Nan GL, Zhai J, Arikit S, Morrow D, Fernandes J, Mai L, Nguyen N, Meyers BC, Walbot V (2017) MS23, a master basic helix-loophelix factor, regulates the specification and development of the tapetum in maize. Development 144:163-172

Nishida I, Tasaka Y, Shiraishi H, Murata N (1993) The gene and the RNA for the precursor to the plastid-located glycerol-3-phosphate acyltransferase of Arabidopsis thaliana. Plant Mol Biol 21:267-277

Patterson E (1995) Tests of male sterile mutants. Maize Genet Coop Newslett 69:126-128

Petit J, Bres C, Mauxion JP, Tai FW, Martin LB, Fich EA, Joubes J, Rose JK, Domergue F, Rothan C (2016) The glycerol-3-phosphate acyltransferase GPAT6 from tomato plays a central role in fruit cutin biosynthesis. Plant Physiol 171:894-913

Pollard M, Beisson F, Li Y, Ohlrogge JB (2008) Building lipid barriers: biosynthesis of cutin and suberin. Trends Plant Sci 13:236-246

Shi J, Cui M, Yang L, Kim YJ, Zhang D (2015) Genetic and biochemical mechanisms of pollen wall development. Trends Plant Sci 20:741-753

Shi J, Gao H, Wang H, Lafitte HR, Archibald RL, Yang M, Hakimi SM, Mo H, Habben JE (2017) ARGOS8 variants generated by CRISPR-Cas9 improve maize grain yield under field drought stress conditions. Plant Biotechnol J 15:207-216

Shockey J, Regmi A, Cotton K, Adhikari N, Browse J, Bates PD (2016) Identification of Arabidopsis GPAT9 (At5g60620) as an essential gene involved in triacylglycerol biosynthesis. Plant Physiol 170:163-179

Skibbe DS, Fernandes JF, Medzihradszky KF, Burlingame AL, Walbot V (2009) Mutator transposon activity reprograms the transcriptomes and proteomes of developing maize anthers. Plant J 59:622-633

Somaratne Y, Tian Y, Zhang H, Wang M, Huo Y, Cao F, Zhao L, Chen $\mathrm{H}$ (2017) ABNORMAL POLLEN VACUOLATION1 (APV1) is required for male fertility by contributing to anther cuticle and pollen exine formation in maize. Plant J 90:96-110

Tan Y, Li S, Xie H, Duan S, Wang T, Zhu Y (2011) Genetical and molecular analysis reveals a cooperating relationship between cytoplasmic male sterility- and fertility restoration-related genes in Oryza species. Theor Appl Genet 122:9-19

Tang JH, Fu ZY, Hu YM, Li JS, Sun LL, Ji HQ (2006) Genetic analyses and mapping of a new thermo-sensitive genic male sterile gene in maize. Theor Appl Genet 113:11-15

Timofejeva L, Skibbe DS, Lee S, Golubovskaya I, Wang R, Harper L, Walbot V, Cande WZ (2013) Cytological characterization and allelism testing of anther developmental mutants identified in a screen of maize male sterile lines. G3 (Bethesda) 3:231-249

Trimnell MR, Patterson E, Fox TW, Bedinger P, Albertsen MC (1999) New chromosome $2 \mathrm{~L}$ male-sterile mutant ms 33 and alleles. Maize Genet Coop Newslett 73:48-49

Vernoud V, Laigle G, Rozier F, Meeley RB, Perez P, Rogowsky PM (2009) The HD-ZIP IV transcription factor OCL4 is necessary for trichome patterning and anther development in maize. Plant J 59:883-894
Wan XY, Wan JM, Jiang L, Wang JK, Zhai HQ, Weng JF, Wang HL, Lei CL, Wang JL, Zhang X, Cheng ZJ, Guo XP (2006) QTL analysis for rice grain length and fine mapping of an identified QTL with stable and major effects. Theor Appl Genet 112:1258-1270

Wan X, Weng J, Zhai H, Wang J, Lei C, Liu X, Guo T, Jiang L, Su N, Wan J (2008) Quantitative trait loci (QTL) analysis for rice grain width and fine mapping of an identified QTL allele gw-5 in a recombination hotspot region on chromosome 5 . Genetics 179:2239-2252

Wang CJ, Nan GL, Kelliher T, Timofejeva L, Vernoud V, Golubovskaya IN, Harper L, Egger R, Walbot V, Cande WZ (2012) Maize multiple archesporial cells 1 (mac1), an ortholog of rice TDL1A, modulates cell proliferation and identity in early anther development. Development 139:2594-2603

Wang DX, Skibbe DS, Walbot V (2013) Maize Male sterile 8 (Ms8), a putative $\beta$-1,3-galactosyltransferase, modulates cell division, expansion, and differentiation during early maize anther development. Plant Reprod 26:329-338

Wang P, Zhang J, Sun L, Ma Y, Xu J, Liang S, Deng J, Tan J, Zhang Q, Tu L, Daniell H, Jin S, Zhang X (2018) High efficient multisites genome editing in allotetraploid cotton (Gossypium hirsutum) using CRISPR/Cas9 system. Plant Biotechnol J 16(1):137-150

Williams ME (1995) Genetic engineering for pollination control. Trends Biotech 13:344-349

Woo JW, Kim J, Kwon SI, Corvalan C, Cho SW, Kim H, Kim SG, Kim ST, Choe S, Kim JS (2015) DNA-free genome editing in plants with preassembled CRISPR-Cas9 ribonucleoproteins. Nat Biotechnol 33:1162-1164

Wu Y, Fox TW, Trimnell MR, Wang L, Xu RJ, Cigan AM, Huffman GA, Garnaat CW, Hershey H, Albertsen MC (2016) Development of a novel recessive genetic male sterility system for hybrid seed production in maize and other cross-pollinating crops. Plant Biotechnol J 14:1046-1054

Xing HL, Dong L, Wang ZP, Zhang HY, Han CY, Liu B, Wang XC, Chen QJ (2014) A CRISPR/Cas9 toolkit for multiplex genome editing in plants. BMC Plant Biol 14:327

Yang W, Simpson JP, Li-Beisson Y, Beisson F, Pollard M, Ohlrogge JB (2012) A land-plant-specific glycerol-3-phosphate acyltransferase family in Arabidopsis: substrate specificity, $s n-2$ preference, and evolution. Plant Physiol 160:638-652

Zhang Y, Liang Z, Zong Y, Wang Y, Liu J, Chen K, Qiu JL, Gao C (2016) Efficient and transgene-free genome editing in wheat through transient expression of CRISPR/Cas9 DNA or RNA. Nat Commun 7:12617

Zhang D, Wu S, An X, Xie K, Dong Z, Zhou Y, Xu L, Fang W, Liu S, Liu S, Zhu T, Li J, Rao L, Zhao J, Wan X (2018) Construction of a multi-control sterility system for a maize male-sterile line and hybrid seed production based on the ZmMs7 gene encoding a PHD-finger transcription factor. Plant Biotechnol J 16:459-471

Zheng Z, Xia Q, Dauk M, Shen W, Selvaraj G, Zou J (2003) Arabidopsis AtGPAT1, a member of the membrane-bound glycerol3-phosphate acyltransferase gene family, is essential for tapetum differentiation and male fertility. Plant Cell 15:1872-1887

Zhu SQ, Zhao H, Zhou R, Ji BH, Dan XY (2009) Substrate selectivity of glycerol-3-phosphate acyl transferase in rice. J Integr Plant Biol 51:1040-1049 